\title{
Erratum: Phase-Matching Quantum Key Distribution [Phys. Rev. X 8, 031043 (2018)]
}

\author{
Xiongfeng Ma, Pei Zeng, and Hongyi Zhou
}

(Received 10 April 2019; published 28 May 2019)

DOI: 10.1103/PhysRevX.9.029901

We found an error in the simulation code in our recent work. With the coding error fixed, the simulation result (Fig. 3 in the original paper) is shown below. The longest distance under the same parameters in Table I is $418 \mathrm{~km}$.

As is shown in Fig. 1, the key rate line of PM-QKD can still overcome the secret key capacity bound when $l>250 \mathrm{~km}$, and the rate-loss dependence of $\mathrm{PM}-\mathrm{QKD}, R \sim O(\sqrt{\eta})$, is not affected.

The simulation error was brought to our attention when we ran the simulation in a follow-up work, and it was independently pointed out by Ivan Djordjevic. In the Supplemental Material [1], we present the MATLAB code of the PM-QKD key rate for reference.

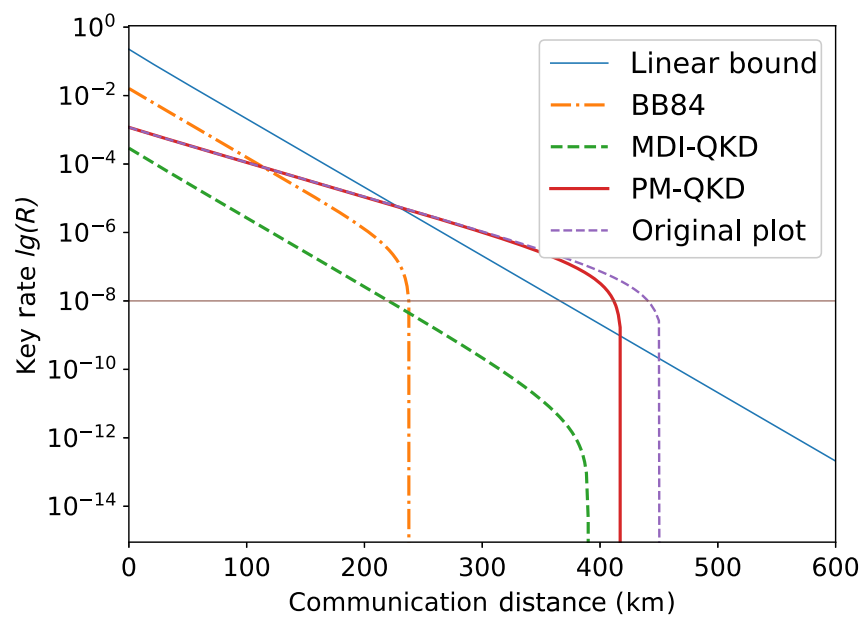

FIG. 1. Corrected simulation result of Fig. 3 in the original article. The purple dashed line is the original incorrect plot of the PM-QKD key rate result, which is caused by an erroneous extra term $(2 \pi) / M$ when calculating the QBER using Eq. (B22); see lines 27 and 28 in the MATLAB code file in the Supplemental Material [1].

[1] See Supplemental Material at http://link.aps.org/supplemental/10.1103/PhysRevX.9.029901 for the MATLAB code of the PM-QKD key-rate evaluation.

Published by the American Physical Society under the terms of the Creative Commons Attribution 4.0 International license. Further distribution of this work must maintain attribution to the author(s) and the published articles title, journal citation, and DOI. 